\title{
Erratum to: Genome sequence of Vibrio diabolicus and identification of the exopolysaccharide HE800 biosynthesis locus
}

David Goudenège • Vincent Boursicot • Typhaine Versigny • Sandrine Bonnetot • Jacqueline Ratiskol • Corinne Sinquin • Gisèle LaPointe - Frédérique Le Roux • Christine Delbarre-Ladrat

Published online: 11 November 2014

(C) Springer-Verlag Berlin Heidelberg 2014

\section{Erratum to: Appl Microbiol Biotechnol}

DOI 10.1007/s00253-014-6086-8

The original version of this article inadvertently contained mistake.

The name of the author F. L. Roux should be presented as F. Le Roux.

The online version of the original article can be found at http://dx.doi.org/ 10.1007/s00253-014-6086-8.

V. Boursicot $\cdot$ T. Versigny $\cdot$ S. Bonnetot $\cdot$ J. Ratiskol $\cdot$ C. Sinquin $\cdot$

C. Delbarre-Ladrat $(\square)$

Ifremer, Laboratoire EM3B (Ecosystèmes Marins et Molécules

Marines pour les Biotechnologies), Rue de l'Ile d'Yeu, BP21105,

F-44311 Nantes Cedex 3, France

e-mail: christine.delbarre.ladrat@ifremer.fr

D. Goudenège $\cdot$ F. Le Roux

Ifremer, Unité Physiologie Fonctionnelle des Organismes Marins, ZI

de la Pointe du Diable, CS 10070, F-29280 Plouzané, France

D. Goudenège $\cdot$ F. Le Roux

Sorbonne Universités, UPMC Univ Paris 06, UMR 8227, Integrative

Biology of Marine Models, Station Biologique de Roscoff, CS

90074, F-29688 Roscoff Cedex, France

D. Goudenège $\cdot$ F. Le Roux $(\bowtie)$

CNRS, UMR 8227, Integrative Biology of Marine Models, Station

Biologique de Roscoff, CS 90074, F-29688 Roscoff Cedex, France

e-mail: frederique.le-roux@sb-roscoff.fr

G. LaPointe

Université Laval, Institute for Nutrition and Functional Foods, 2440

boul. Hochelaga, Québec, Québec, Canada G1V 0A6 